\title{
AN OPEN-SOURCE POPULATION INDIFFERENCE ZONE-BASED ALGORITHM FOR SIMULATION OPTIMIZATION
}

\author{
Theodore T. Allen \\ David N. Vuckovich \\ The Ohio State University \\ Dept. of Integrated Systems Engineering \\ 1971 Neil Avenue - 210 Baker Systems \\ Columbus, OH 43210, USA
}

\begin{abstract}
This paper proposes an open-source algorithm for simulation optimization. The intent is to permit many who use a variety of simulation software codes to be able to apply the proposed methods using an MS Excel-Visual Basic interface. First, we review selected literature on simulation optimization and its usefulness. Then, we briefly discuss methods that are commonly used for simulation optimization. Next, we present the proposed Population Indifference Zone (PIZ) algorithm and related software code. Also, we discuss the properties of the proposed method and present the code that runs the Visual Basic program. Finally, we discuss the functionality of the Population Indifference Zone method with examples of problems to which it might be applied and conclude with topics for future research.
\end{abstract}

\section{INTRODUCTION}

Discrete event simulation continues to grow in popularity presumably because it helps increase profitability through the avoidance of problems in business expansion and manufacturing. Simulation allows for the testing of how system parameters (factors) impact system performance (responses) without costly and time consuming testing on the actual systems. Simulation optimization is the systematic search for factor settings that derive the most desirable expected response values. Interest in simulation optimization also continues to grow perhaps because increasing computer power makes solving large simulation optimization problems computationally feasible (Zhao and Sen 2006).

More generally, after a simulation code has been built and validated, it is then studied to provide decision-support. These phases are often called "output analysis" and generally involve some form of optimization (Fu, Glover, and April 2005). As a motivating example, consider a problem at a major automotive manufacturer that is evaluating alternative policies for running a major production line. Examples of factors in the simulation optimization study include work-in-process management policy (off or on), the conveyor speed at the wash substation, and the transfer time for units at a major substation. By varying these factor settings, a large number of alternative systems are developed and studied using the simulation codes. Because each evaluation is noisy, there is generally a concern about how many replicates of each run should be used to gain useful information and (hopefully) derive the optimal system or combination of factor settings.

Simulation optimization methods can be divided into two types: (1) problem specific and (2) generic or "black box" methods (Zhao and Sen 2006). The problem specific methods usually require less time to find a solution, but are generally more difficult to apply in simulation optimization because they require 


\section{Allen and Vuckovich}

the attention of expert operations researchers. Sometimes problem generic methods can be relatively inefficient because they involve comparison of solutions that give very similar average response values. Such comparisons are computationally costly particularly when each simulation takes a long time ( $\mathrm{Fu}$, $\mathrm{Chen}$, and Shi 2008). On the other hand, black box methods can be applied to many types of problems and can be used by anyone with limited knowledge of simulation.

Black box methods can further be divided into methods based on constant sample sizes and systemically varying sample sizes. There are multiple types of black box methods including tabu searches, scatter searches, and genetic algorithms. In the context of constant sample size methods, tabu searches and scatter searches have been established as the most effective according to many authors ( $\mathrm{Fu}$, Glover, and April 2005). The other simulation optimization methods being employed and studied are ranking and selection, response surface methodology, gradient-based procedures, stochastic approximation, random search, and sample path optimization (Fu, Glover, and April 2005; Fu, Chen, and Shi 2008).

For the variable sample size, black box methods, the computational dominance of black box and scatter search methods is less well established. Here, we focus on hybrid technology that involves a population search method (like a genetic algorithm) combined with selection and ranking methods. Therefore, this paper follows up on the essential insights in Boesel, Nelson, and Ishii (2003). However, the methods here have rigorous properties building on mathematical results in Zheng and Allen (2007).

Many commercial codes support optimization using simulation through either full factorial experimental designs or through deterministic heuristics applied to simulation optimization. In either case, these methods historically tended to use constant numbers of samples for each solution being evaluated at each iteration in the method. However, recently most of the main simulation software, such as Arena, AnyLogic, ProModel and its affiliated software, FlexSim, and SIMUL8 use OptQuest as the optimizer for their simulations (Swain 2007; Rockwell Automation 2009; Fu, Glover, and April 2005; ProModel 2009). OptQuest is a "general-purpose optimizer" which runs a complicated metaheuristic using a variable sample size scatter search and tabu search to find optimal solutions (Laguna 1997; Fu, Glover, and April 2005). The method of ranking and selection has become increasingly studied and applied in order to come to conclusions on the goodness of a solution (Fu, Chen, and Shi 2008).

It is likely that OptQuest offers greater computational efficiency than the methods here as well as better integration with existing software packages. However, we are not aware of any rigorous properties related to convergence or other aspects associated with OptQuest. The intent here is to offer open-source code for a method with likely reasonable performance that is associated with rigorous convergence properties. Therefore, the proposed method can be viewed as a springboard for more efficient methods. Also, for cases in which integration with OptQuest or its cost are prohibited, the proposed methods may fill a useful gap.

Section 2 proposes the population indifference zone (PIZ) method. In Section 3, rigorous properties of the PIZ method are established building on results in Zheng and Allen (2007). Section 4 describes details related to implementing the code which can be downloaded from the web. Problems that can be used to compare the proposed method with alternatives are described in Section 5. Section 6 closes with a brief discussion and description of opportunities for future research.

\section{THE POPULATION INDIFFERENCE ZONE (PIZ) METHOD}

In this section, the population indifference zone (PIZ) method is proposed. The method includes three sub-phases: the formation of the solutions to be evaluated (e.g., creation of a new population in a genetic algorithm), the preliminary evaluation of those solutions using subset selection, and the follow-up evaluation of solutions (if necessary) using an indifference zone procedure.

The specific problem that this paper is aimed at solving is: minimize $E(y(x i)+\varepsilon)$.

Subset selection (steps 2-5) is described in Goldsman, Nelson, Opicka, and Pritsker (1999). That procedures and those steps achieve a subset having a probability greater than $P^{*}$ of containing a solution with mean or expected value within $\delta_{(1)}$ of the optimal or best system solution. The indifference parameter, $\delta_{(1)}$, can be set to zero, but generally fewer systems will be eliminated. Let $k$ denote the number of alternative 


\section{Allen and Vuckovich}

systems being compared. Assume we want to find a subset containing a system with mean within $\delta_{(1)}$ of the smallest, i.e., we are minimizing.

The indifference zone method is then used (steps 6-9) which is a two-stage selection from Sullivan and Wilson (1989). The sub-procedure starts with a set of $k$ alternative systems and terminates with a subset of $m$, where the method user picks $m$. The user picks $m$ together with the indifference parameter $\delta_{2}$ and the lower bound on the quality probability $P^{*}$. For example, one might start with $k=100$ systems and plan to end with 10 systems with one having a mean within $\delta_{(2)}=3.0$ of the true best mean from the original 100 with probability $P^{*}=0.95$. The procedure is based on pre-tabulated Rinott's constants, denoted here as $h=\operatorname{Rinott}_{k, m, n 0, P^{*}}$. Rinott (1978) was probably the first to tabulate these constants.

\section{Population Indifference Zone (PIZ) Method}

Step 1. (Initialization) Create $k$ system alternatives by sampling uniformly from the decision-space.

Step 2. Evaluate all systems using $n_{0}$ samples, which are generally batch sample average values. Denote the resulting values $X_{i, j}$, with $i=1, \ldots, k$ referring to the system and $j=1, \ldots, n_{0}$ referring to the sample.

Step 3. Calculate the sample means or Monte Carlo estimates, $X_{\mathrm{bar}, i}$, for each system $i=1, \ldots, k$. Denote the index for the system with the best mean as " $b$ ".

Step 4. Next, we examine the differences between the samples from each system paired with the samples from system $b$. Compute the standard deviations of the differences using:

$S_{i, j}^{2}=\left\{\sum_{j=1, \ldots, n 0}\left[X_{i, j}-X_{b, j}-\left(X_{i, \mathrm{bar}}-X_{b, \mathrm{bar}}\right)\right]^{2}\right\} /\left(n_{0}-1\right)$

and

$W_{i, b}=\left(t_{v, n 0-1}\right)\left(S_{i, b}\right) /\left[\left(n_{0}\right)^{1 / 2}\right]$

with

$v=1-\left(P^{*}\right)^{[1 /(k-1)]}$

and where " $t$ " is a critical value for the t-distribution with $\alpha=v$ and $n 0-1$ degrees of freedom.

Step 5. Form the subset by including only the systems with means satisfying:

$$
\left(X_{i, \text { bar }}-X_{b, \text { bar }}\right) \leq \operatorname{maximum}\left(W_{i, b}-\delta_{(1)}, 0.0\right)
$$

where the "maximum" implies taking the larger value which could be 0.0 . If the subset contains less than $10 \%$ of the generation skip to step 10 .

Note that the above method with $\delta=0$ is essentially creating single sample differences of each subsystem and the apparently best system. The approach essentially creates these differences and t-tests whether the differences are statistically significant. With $\delta=0$, the previous steps are guaranteed to keep the best solution with probability greater than $P^{*}$.

Step 6. (First stage, optional because we can reuse the Step 2 evaluations) Evaluate all systems with $n_{0}$ samples. These are generally batch means with typical initial values equal to $n_{0}=10$ or $n_{0}=20$. Fewer samples are generally needed if the batches are large. Calculate the sample means, $X_{i, \text { bar, }}$, and sample standard deviations, $S_{i}$, for all system responses.

Step 7. Calculate the total number of samples first stage plus follow-up for each system using:

$n_{i}=\operatorname{maximum}\left\{n_{0}+1, \operatorname{roundup}\left[(h)^{2}\left(S_{i}\right)^{2} /\left(\delta_{(2)}\right)^{2}\right]\right\}$.

Step 8. (Second stage) Perform the additional $n_{i}$ runs and then calculate the means of these second stage runs, $\mathrm{X}_{\mathrm{bar}, i}{ }^{(2)}$. Denote the index for the system with the best mean as " $b$ ".

Step 9. Using the first stage standard deviations, $S_{i}$, calculate: 


$$
W_{i}=\frac{n}{n_{i}}\left[1+\sqrt{1-\frac{n_{i}}{n}\left(1-\frac{\left(n_{i}-n\right) \delta_{(2)}^{2}}{h^{2} S_{i}^{2}}\right)}\right] \text { for } i=1, \ldots, k
$$

and keep $m$ subsystems with among these with the smallest $W_{i} X_{i, \text { bar }}+\left(1-W_{i}\right) \mathrm{X}_{i, b a r}{ }^{(2)}$ values.

The above procedure (Steps 6-9) is essentially the same as the Koenig and Law (1985) procedure.

Step 10. (Termination) Is any of our solutions good enough? If yes, stop, otherwise continue. Or if stopping rule has been reached, stop, otherwise continue.

Step 11. (Form the next population) Copy the $10 \%$ highest ranked solutions into the next population. If the subset selection eliminated $90 \%$ or more solutions, the ones left are the elitist subset. Otherwise, they are the subset from the indifference zone procedure. Fill in $80 \%$ of the total generation by selecting two systems at random, then for each variable in the system select a random number from 0 to 1 . If the number is greater than .8 switch the level of the selected variable of the two systems. Repeat this for each variable. Fill the remaining $10 \%$, or slightly more depending on the results of the subset selection, solutions with uniform random selections from the decision-space.

Wilcox (1984) presents tables for Rinott's constants including those in Table 1. Use the condensed table below to select the appropriate Rinott's constant $h$. This is for use when population size $k$ is equal to 10 and where $l$ is the number of solutions in the subset. When we use 100 solutions in the population and $n_{0}$ equal to 10 the bound on $h$ is 3.3 . We will use this as an approximation in this code because we do not currently have a proper lookup function to find all possible Rinott's constants.

Table 1: Rinott's constant values for population of size 10 where " $l$ " is the \# of solutions not eliminated in subset selection.

\begin{tabular}{|c|c|}
\hline$k$ & 10 \\
\hline$n_{0}$ & 5 \\
\hline$P^{*}$ & .95 \\
\hline$l$ & $h$ \\
\hline 2 & 3.107 \\
\hline 3 & 3.908 \\
\hline 4 & 4.390 \\
\hline 5 & 4.746 \\
\hline 6 & 5.025 \\
\hline 7 & 5.260 \\
\hline 8 & 5.463 \\
\hline 9 & 5.641 \\
\hline 10 & 5.799 \\
\hline
\end{tabular}

The above method can be used very generally as long as solutions can be sampled uniformly from the solution space. It essentially draws from the huge space of solutions batches and then compares the batches efficiently using selection and ranking methods. In the next section, some of the rigorous properties of the PIZ method are established.

The presented PIZ method is only a framework in the sense that virtually any method could be used to fill the population including tabu and scatter search methods. The only step that would be modified would be Step 11 to the specifications of the method chosen to fill the population. 


\section{Allen and Vuckovich}

\section{METHOD PROPERTIES}

There are multiple desirable properties of the PIZ method. The first is that it is a black box method in the way that it is relatively simple and can be applied to virtually all simulation optimization problems as long as one can sample uniformly from the search space. The second property is that it offers some potential advantage in computational efficiency compared with constant sample size methods. This follows because poor quality solutions are usually removed from consideration in Step 5. This way, these solutions do not waste valuable CPU run time during the relatively exhaustive indifference zone-based evaluations in Step 8.

In addition, the PIZ method is associated with some rigorous claims about the solution quality as we next establish. This is not true for other methods of similar type including the genetic algorithm-based methods in Aizawa and Wah (1994) and Bernshteyn (2001). That makes it perhaps a strong platform for additional method development.

The first rigorous claim is described in Theorem 1.

Theorem 1. Assume that there are $N$ normal populations in the search space ( $N$ is generally very large). After $M$ iterations, the PIZ method will retain at least one solution with objective value within $(M \times \delta)$ of the best solution evaluated with probability greater than $\left(P^{*}\right)^{2 M}$.

Proof. In the subset selection steps, we keep the best solution with probability greater than $P^{*}$. In the indifference zone steps we keep a solution within $\delta$ of the best with probability greater than $P^{*}$. Therefore, in each complete iteration we keep a solution within $\delta$ of the best with probability greater than $\left(P^{*}\right)^{2}$. This follows because of independence of the two positive events, i.e., keep the best and then keep a solution within $\delta$ of the best. In the next iteration, there might be an additional loss of $\delta$. Therefore, after $M$ iterations the loss is guaranteed to be less than $(M \times \delta)$ with probability greater than $\left(P^{*}\right)^{2 M}$.

Zheng and Allen (2007) describes the changing of both the $\delta$ and the $\mathrm{P}^{*}$ in each population as the algorithm iterates. They do this to provide long run guarantees based on essentially Theorem 1. Examples of schedules offering long run guarantees are in equations (7), (8), (9), and (10). If at each iteration one has the probability of greater than $P_{t}$, to keep the solution with an objective value within $\delta_{t}$ of the real best solution in generation $t$, then:

$$
\begin{aligned}
& P^{*}=\prod_{t=0}^{\infty} P^{t}, \text { and } \\
& \Delta=\sum_{t=0}^{\infty} \delta_{t}
\end{aligned}
$$

Using the above equations one can calculate $\delta_{t}$ and $P^{t}$ for each generation $\mathrm{t}$ using the final $\Delta$ and $P^{t}$ chosen by the user. These equations are presented below where $\epsilon, \mathrm{o}, \mathrm{u}$, and $\mathrm{s}$ are predetermined parameters where $0<\mathrm{s}<1, \mathrm{o}<\mathrm{u}<1$ and $0<\epsilon<1$.

$$
\begin{aligned}
& \delta_{t}=\Delta(1-s) s^{t} \\
& P^{t}=1-\frac{u}{(t+o)^{1+\varepsilon}}
\end{aligned}
$$

Applying a schedule to $\mathrm{dt}$ and $\mathrm{pt}$ can generate long run convergence to within $\mathrm{d}$ of global optimum with probability $\mathrm{P}^{*}$.

\section{CODE DETAILS}

\subsection{Code explanation}

The MS Excel-Visual Basic code uses a user-form to receive information from the user like number of variables, generation size, and total generations. The code itself breaks down into 6 main sections: setup, 


\section{Allen and Vuckovich}

mapping, subset selection, indifference zone, cross-over, and output. In the setup basic computer programming techniques are applied: variables are named and their data type defined, arrays are dimensioned, and some variable values are set. The mapping section, which could possibly present the most challenging issue when trying to solve some problems, selects random variables from a uniform $(0,1)$ distribution and maps them to the decision-space, which for the A4 function described in section 5.1 and used in this code is $(-1.28,1.28)$. The function used to do this is $x=1.28-2.56[\mathrm{~N}(0,1)]$. It is irrelevant that $x$ will always be negative because $x^{4}$ is always positive.

In general, the code uses many for loops and arrays to store the information needed. In the subset selection section the objective value of each iteration of each system is calculated to then find $X_{b a r}$. Application.Worksheetfunction. Small is used to find the smallest objective value $X_{\text {bar }}$. Before the systems are selected the code finds the number that will be selected so that an array Copyover() can be dimensioned properly. Once the subset is selected it moves on to the indifference zone. First it checks to see if Copyover() has more than $10 \%$ of the original population in it. If it does it proceeds. The indifference zone is rather similar to the subset selection section, but with slightly different computations. The major difference is that in the indifference zone section some systems require more samples than others. To account for this we found the largest new sample size required. The array was then dimensioned with that $\mathrm{n}$, and each system requires a reference to its new sample size.

The cross-over section begins by selecting the systems at random with uniform probabilities of being chosen by using Application.Worksheetfunction.Ceiling[GenerationSize * Rnd() 1]. This selects a system 1 through the total number in the generation to be one of the parents. We use four new arrays called FirstParent(), SecondParent(), FirstChild(), and SecondChild(). Once the parents are selected a random number $(0,1)$ is picked. If the number is greater than .8 the value for the variable in FirstParent() is assigned to the value for the variable in SecondChild() and vice versa. If the number is less than .8 the value for the variable in FirstParent() is assigned to the value of the variable in FirstChild(). The code uses the basic Cells $(\mathrm{R}, \mathrm{C})$ command to output the value of the variables from the selected system.

\subsection{Adapting the code}

If one were interested in adapting the code to another function, all that would be necessary is to change the mapping section of the code. This is where the A4 function is located in the code. The comment in the code says, "Runs the A4 problem as a simulation." Filling the population could be changed as well, but would take more effort. All that needs to be done is change out the cross-over section and write code for tabu search or scatter search or your favorite search method.

\section{NUMERICAL EXAMPLES}

In this section, problems are described that can be used to evaluate the PIZ method and compare its computational performance with alternatives. These include the A4 function and the noisy Rastrigin function.

\subsection{A4 function}

Aizawa and Wah (1994) and Bernshteyn (2001) use the A4 test function. This function is built into the code. This test function involves the random variable $\varepsilon$ which is $\mathrm{N}(0,1)$ and can be written:

$$
\mathrm{y}(x)=\left[\sum_{i=1}^{k} i x_{i}^{4}\right]+A \epsilon
$$

where typically $k=30$ dimensions and $-1.28 \leq x \leq 1.28$. This equation can be studied for different values of $A$ from 1 to 64, do 10 replicates of the whole procedure.

The PIZ method was tested with the A4 function. The results are shown in Tables $2 \& 3$ and graphical representations are shown in Figures $1-4$. The solution strength was measured by evaluating the A4 function without noise. Y-Bar is the average of four of these replicates. After seeing these results one can be confident in saying that the PIZ method is superior to constant sample size, especially for more va- 


\section{Allen and Vuckovich}

riables (10) in a smaller population (10). It is also clear everything but sigma had a major impact on the average objective value; however, if sigma were varied more than just 1 there would probably have been a larger impact. We can say this just from experience while debugging the code. This would be another issue to address in the future. The total number of simulations run had a smaller effect than the other factors.

Table 2: Results of PIZ Method Testing for the A4 Function.

\begin{tabular}{|r|rr|r|r|r|c|}
\multicolumn{1}{c}{ A } & \#Variables & \multicolumn{1}{c}{$n_{0}$} & Generation Size & \# Function Evaluations & Y_Bar & S \\
\hline 2 & 3 & 5 & 10 & 1000 & 0.1789 & 0.3024 \\
\hline 2 & 3 & 5 & 10 & 10000 & 0.6371 & 0.4811 \\
\hline 2 & 10 & 5 & 10 & 1000 & 5.3653 & 3.6542 \\
\hline 2 & 10 & 5 & 10 & 10000 & 3.3782 & 1.8258 \\
\hline 2 & 3 & 10 & 100 & 1000 & 0.0094 & 0.0071 \\
\hline 2 & 3 & 10 & 100 & 10000 & 0.0141 & 0.0154 \\
\hline 2 & 10 & 10 & 100 & 10000 & 4.2584 & 1.0010 \\
\hline 2 & 10 & 10 & 100 & 1000 & 0.1424 & 0.1677 \\
\hline 1 & 3 & 5 & 10 & 10000 & 0.1919 & 0.1978 \\
\hline 1 & 3 & 5 & 10 & 1000 & 6.3125 & 1.5192 \\
\hline 1 & 10 & 5 & 10 & 10000 & 1.5610 & 0.7929 \\
\hline 1 & 10 & 5 & 10 & 1000 & 0.0171 & 0.0181 \\
\hline 1 & 3 & 10 & 100 & 10000 & 0.0054 & 0.0055 \\
\hline 1 & 3 & 10 & 100 & 1000 & 3.8757 & 1.2707 \\
\hline 1 & 10 & 10 & 100 & 1.4220 & 0.6787 \\
\hline 1 & 10 & 10 & 100 & & & \\
\hline
\end{tabular}

Table 3: Results of Constant Size Method for the A4 Function.

\begin{tabular}{|r|r|r|r|r|r|c|}
\multicolumn{1}{c}{ A } & \#Variables & \multicolumn{1}{l}{$n_{0}$} & Generation Size & \# Function Evaluations & Y_Bar & S \\
\hline 2 & 3 & 20 & 10 & 1000 & 1.2510 & 1.1176 \\
\hline 2 & 3 & 20 & 10 & 10000 & 0.1972 & 0.2159 \\
\hline 2 & 10 & 20 & 10 & 1000 & 16.0049 & 8.0635 \\
\hline 2 & 10 & 20 & 10 & 10000 & 14.6057 & 4.2168 \\
\hline 2 & 3 & 20 & 100 & 1000 & 0.0108 & 0.0097 \\
\hline 2 & 3 & 20 & 100 & 10000 & 0.0245 & 0.0153 \\
\hline 2 & 10 & 20 & 100 & 1000 & 3.5343 & 2.4640 \\
\hline 2 & 10 & 20 & 100 & 1000 & 3.1535 & 0.8398 \\
\hline 1 & 3 & 20 & 10 & 10000 & 0.8605 & 0.5932 \\
\hline 1 & 3 & 20 & 10 & 1000 & 17.2263 & 0.9407 \\
\hline 1 & 10 & 20 & 10 & 10000 & 13.3052 & 6.2762 \\
\hline 1 & 10 & 20 & 10 & 1000 & 0.0476 & 0.0396 \\
\hline 1 & 3 & 20 & 100 & 10000 & 0.0201 & 0.0352 \\
\hline 1 & 3 & 20 & 100 & 1000 & 5.0307 & 2.4679 \\
\hline 1 & 10 & 20 & 100 & 10000 & 3.4815 & 1.8323 \\
\hline 1 & 10 & 20 & 100 & & & \\
\hline
\end{tabular}




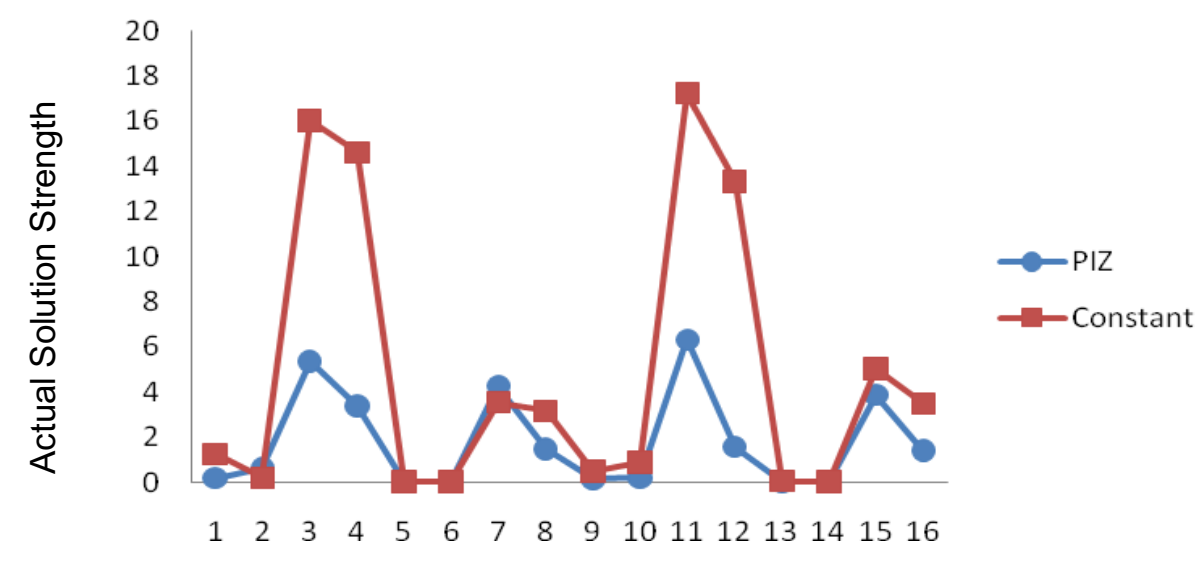

Figure 1: A graph of solution strength comparing the PIZ method and constant sample size.

\section{Sample Stand. Dev. PIZ vs. Constant Sample Size}

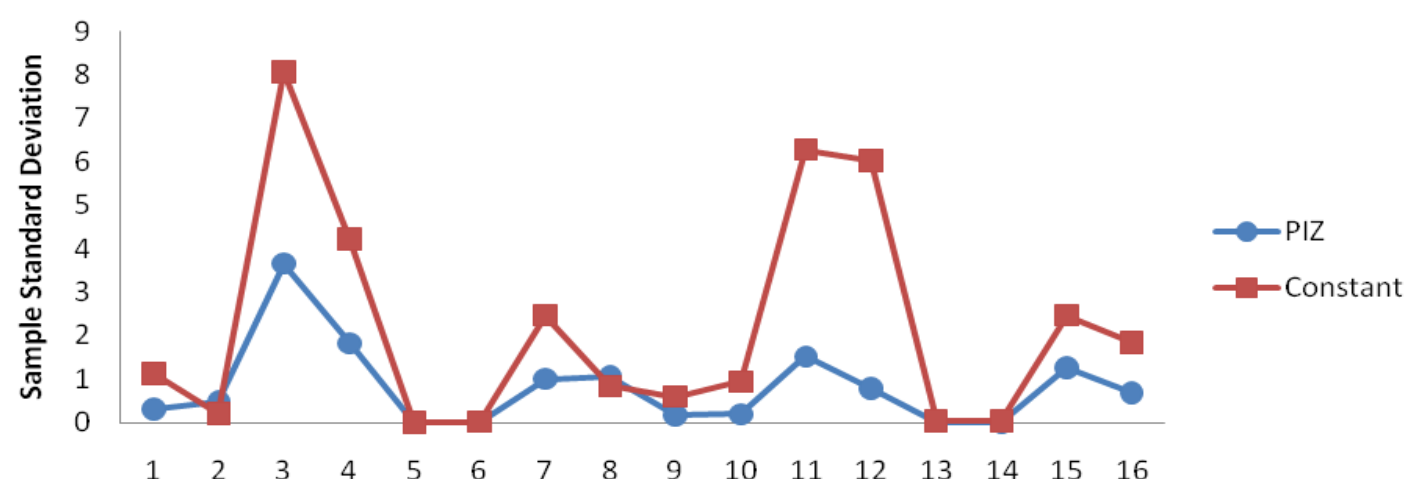

Figure 2: A graph of sample standard deviation comparing the PIZ method and constant sample size.

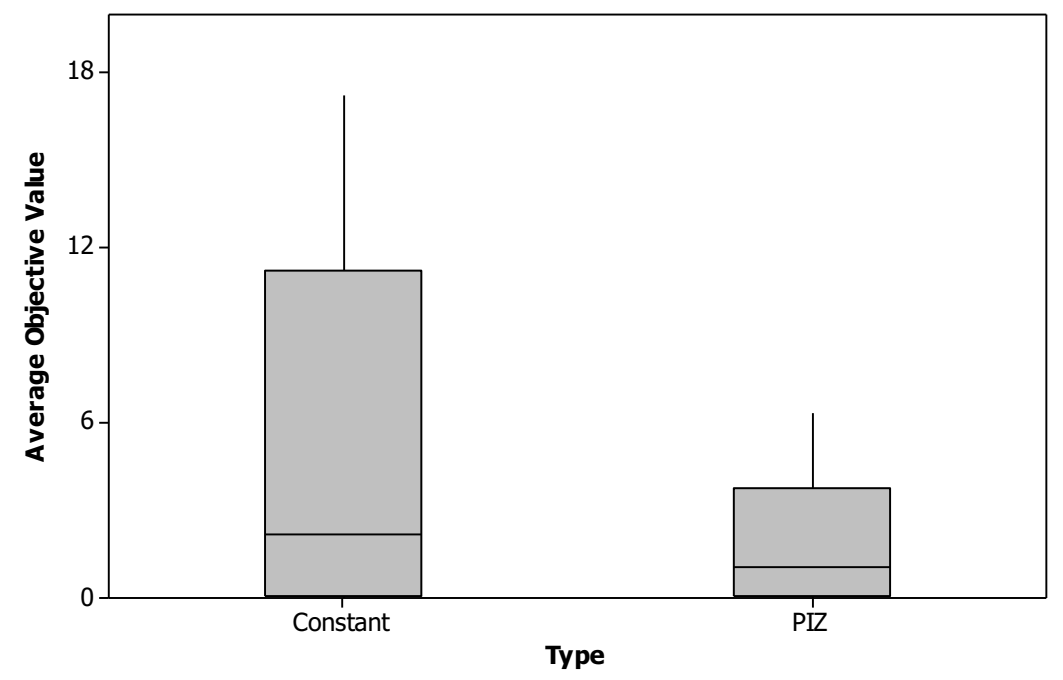

Figure 3: Box plot of the average objective value (solution strength) of constant sample size and PIZ. 
Main Effects Plot (data means) for Average Objective Value

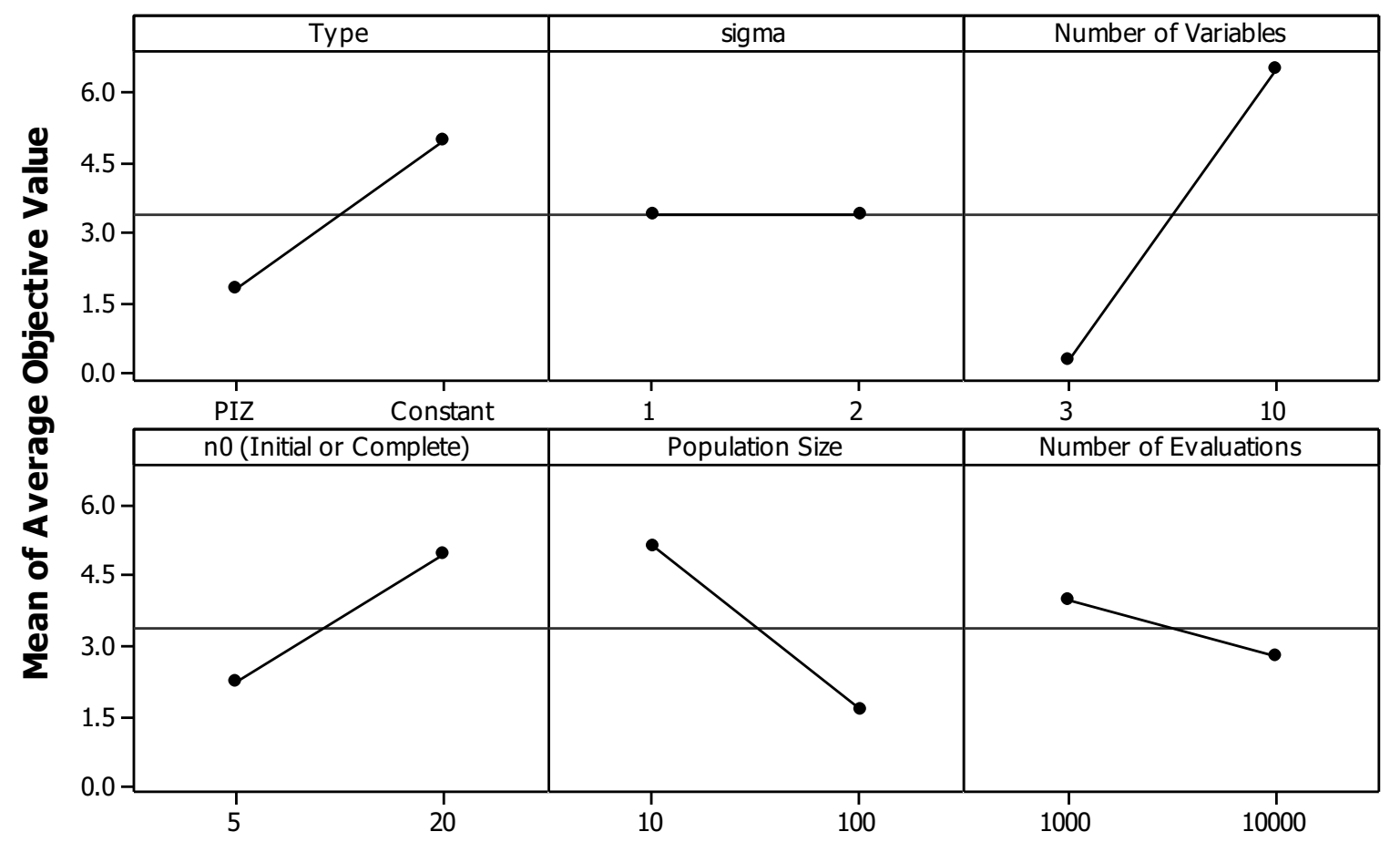

Figure 4: Main effects plot for the average objective value (solution strength).

\section{DISCUSSION}

This paper has proposed an algorithm for simulation optimization called the population indifference zone (PIZ) method. It is called an algorithm because it is associated with rigorous convergence guarantees as described in section 3. Perhaps more importantly, it is available by corresponding with the authors or possibly through other means as well with open-source in a Visual Basic implementation. There are, however, a number of opportunities for future research.

First, the PIZ method can be compared computationally with constant sample size and other alternatives using the functions in Section 5. Such results can establish the benefits of using the method in practice. As for now it is clear that that PIZ method is superior to the constant sample size method. The robustness of the PIZ method is still in question and will soon be tested with the Rastrigin function. Second, a download site for the code can be established to facilitate others benefiting from the code. Third, an example of using SHELL of other visual basic commands to control an actual simulation code executable can be developed. This will help engineers at major companies benefit from simulation optimization even in cases in which connection with OptQuest is difficult or impossible.

Finally, the application of PIZ to a real problem could illustrate the method and its practical benefits. This could be done in an integrated method together with other visual basic capabilities at a major manufacturer.

\section{ACKNOWLEDGMENTS}

This work was partially supported by a grant from Honda of America. John Gillard, Kirby Clark, Jason Petrinowitsch, and Timothy Leopold provided insights into what matters in real-world applications. Also, 


\section{Allen and Vuckovich}

we would like to thank Cathy Xia for agreeing to be on the committee. We would also like to thank our parents for their unending support along with our friends.

\section{REFERENCES}

Aizawa, A.N., and B.W. Wah, 1994. Scheduling of genetic algorithms in a noisy environment. Evolutionary Computation 2(2):97-122.

Rockwell Automation 2009. Arena comparison statement. Available via: <http: / / www . arenasimulation. com/news / docs /Arena\%20 comparison\%20state ment $\div 202007$.pdf $>$ [accessed April 14, 2010].

Bernshteyn, M. 2001. Simulation optimization methods that combine multiple comparisons and genetic algorithms with applications in design for computer and supersaturated experiments. Ph.D. Thesis, The Ohio State University, Industrial, Welding \& Systems Engineering.

Boesel, J., B. L. Nelson, and N. Ishii. 2003. A framework for simulation-optimization software. IIE Transactions 35(3):221-229.

Fu, M. C., C.-H. Chen, and L. Shi. 2008. Some topics for simulation optimization. In Proceedings of the 2008 Winter Simulation Conference, eds. S. J. Mason, R. R. Hill, L. Mönch, O. Rose, T. Jefferson, J. W. Fowler, 27-38. Piscataway, New Jersey: Institute of Electrical and Electronics Engineers, Inc.

Fu, M. C., F. W. Glover, and J. April. 2005. Simulation optimization: a review, new developments, and applications. In Proceedings of the 2005 Winter Simulation Conference, eds. M. E. Kuhl, N. M. Steiger, F. B. Armstrong, and J. A. Joines, 83-95. Piscataway, New Jersey: Institute of Electrical and Electronics Engineers, Inc.

Goldsman D., B. L. Nelson, T. Opicka, and A. A. B. Pritsker. 1999. A ranking and selection project: experiences from a university-industry collaboration. In Proceedings of the 1999 Winter Simulation Conference, eds. P. A. Farrington, H. B. Nembhard, D. T. Sturrock, and G. W. Evans, 83-92. Piscataway, New Jersey: Institute of Electrical Engineers Inc.

Koenig, L. W., and A. M. Law.1985. A procedure for selecting a subset of size $\mathrm{m}$ containing the $\mathrm{i}$ best of $\mathrm{k}$ independent normal populations, with applications to simulation. Communications in Statistics: Simulation and Computation 14:719-734.

Laguna, M. 1997. Optimization of complex systems with OptQuest. Research report, University of Colorado, Graduated School of Business Administration.

ProModel 2009. OptQuest ${ }^{\circledR}$ for ProModel. ProModel Corporation. Available via: <http://www.promodel.com/products/optquest/> [accessed September 9, 2010].

Rinott, Y. 1978. On two-stage selection procedures and related probability inequalities. Communications in Statistics 7: 799-811.

Swain, J.J. 2007. Simulation software survey. ORMS Today 34(5):1-8.

Sullivan, D. A., and J.R. Wilson. 1989. Restricted subset selection procedures for simulation, Operations Research 37(1):52- 71.

Wilcox, R.R. 1984. A table for Rinott's selection procedure. Journal of Quality Technology 16:97-100.

Zhao, L., and S. Sen. 2006. A comparison of sample-path based simulation-optimization and stochastic decomposition for multi-location transshipment problems. In Proceedings of the 2006 Winter Simulation Conference, eds. L. F. Perrone, F. P. Wieland, J. Liu, B. G. Lawson, D. M. Nicol, and R. M. Fujimoto, 238-245. Piscataway, New Jersey: Institute of Electrical and Electronics Engineers, Inc.

Zheng, N., and T. T. Allen. 2007. Subset selection and optimization for selecting binomial systems applied to supersaturated design generation. In Proceedings of the 2007 Winter Simulation Conference, eds. S. G. Henderson, B. Biller, M. -H. Hsieh, J. Shortle, J. D. Tew, and R. R. Barton, 331-339. Piscataway, New Jersey: Institute of Electrical and Electronics Engineers, Inc. 


\section{AUTHOR BIOGRAPHIES}

THEODORE T. ALLEN is an Associate Professor of Integrated Systems Engineering at The Ohio State University. Dr. Allen's research is devoted to the interaction between statistical models, data collection, optimization, and human-computer interaction. He has co-authored or authored over forty peer reviewed publications and two textbooks including a recent textbook on simulation and agent-based modeling intended to help bridge between the classroom and the Winter Simulation Conference. His research on simulating election systems has gained international attention and is featured in his simulation textbook. He is a fellow of the American Society for Quality. His email address is <allen.515@osu .edu>.

DAVID N. VUCKOVICH is a former honors student at The Ohio State University. Currently he is training to become a high school teacher at Wright State University. His email address is <david.vuckovich@gmail.com>. 